\title{
La intervención docente en educación física durante el periodo de cuarentena por COVID-19 Teaching intervention in physical education during quarantine for COVID-19
}

\author{
*Salvador Baena-Morales, **Juan López-Morales, ***Olalla García-Taibo
}

*Universidad de Alicante (España), **Universidad de Granada (España), ***Universidad Pontifica de Comilla (España)

\begin{abstract}
Resumen. El objetivo de este estudio fue describir el cambio producido en la intervención docente del profesorado de Educación Física como consecuencia de la cuarentena por COVID-19. Los datos de la investigación procedieron de una encuesta online realizada a 191 docentes en activo. La encuesta se compuso de 15 preguntas agrupadas en tres dimensiones principales, la reorganización de los contenidos, el análisis de la evaluación y los cambios metodológicos realizados. Los principales resultados del estudio indican que la mayoría de los docentes no han continuado con el horario de docencia previo a la cuarentena. La evaluación se destacó como la dimensión de mayor controversia, existiendo un alto porcentaje de docentes que no tenían previsto como evaluar e incluso no lo veían factible. El bloque de contenidos relacionado con la condición física y la salud fue el más utilizado por el profesorado. Adicionalmente, se describen una serie de dificultades detectadas con la implementación de la enseñanza virtual. Finalmente, la gran mayoría de encuestados consideró que la cuarentena ha podido suponer un problema psicológico y de salud a su alumnados. Estos resultados pueden servir de referencia para futuras investigaciones que se realicen relacionadas con la docencia virtual de la Educación Física.
\end{abstract}

Palabras clave: Coronavirus; Método; Educación; Evaluación; Comunicación.

Abstract. The aim of this study was to describe the changes in teaching intervention in Physical Education because of the quarantine caused by COVID-19. Data was collected from 191 active teachers by an online questionnaire, which consisted of 15 questions grouped into three main dimensions: contents reorganization, evaluation analysis and methodological changes. The main results of this study indicated that most of the teachers did not continue with the teaching hours prior quarantine. Regarding the evaluation, a high percentage of teachers neither planned it nor found it feasible. Contents of Physical condition and health were the most commonly used among teachers. Additionally, several difficulties were detected regarding the implementation of virtual teaching. Finally, the majority of teachers answered that quarantine could involve psychological and health problems for their students. These results could be considered for future research focused on Virtual Physical Education.

Key words: Coronavirus; Method; Education; Evaluation; Communication.

\section{Introducción}

La epidemia provocada por el COVID-19 se ha extendido por todo el mundo convirtiéndose en una pandemia global en poco más de dos meses (Huang et al., 2020). Su impacto se está sufriendo principalmente a nivel sanitario, pero también en los diversos estamentos sociales, como el educativo (Wang et al., 2020). La Organización de la Naciones Unidas para la Educación, la Ciencia y la Cultura (UNESCO) estima que aproximadamente 40 millones de niños no asistirán a la escuela en todo el mundo (Cluver et al., 2020). En España, se ha establecido un estado total de cuarentena, conllevando el cese de la actividad académica presencial. Este problema, sumado al confinamiento general en el hogar, representa una amenaza para la salud de los niños al prescindir de actividades al aire libre y de relaciones sociales con amigos de su edad (Wang et al., 2020). Además, la falta de asistencia a la escuela podría implicar otros perjuicios como la pérdida de habilidades relacionadas con el aprendizaje (Burgess \& Sievertsen, 2020), el descenso del rendimiento académico y la ruptura de la rutina asociada al colegio (Brazendale et al., 2017).

La falta de estructuración del horario y de los hábitos del día a día de los niños debido al confinamiento, pueden derivar en comportamientos obesogénicos. El periodo de cuarentena puede propiciar estímulos alimentarios perjudiciales, sedentarismo e incluso un ambiente estresante (Emery cet al., 2015), sin embargo la escuela promueve la actividad

Fecha recepción: 26-05-20. Fecha de aceptación: 21-07-20

Juan López-Morales

jlopezmorales@ugr.es física, los hábitos de sueño y una dieta apropiada (Brazendale et al., 2017). En particular, la asignatura de Educación Física (EF) representa un papel educativo clave ya que sus contenidos están íntimamente vinculados a la salud, contribuyendo mediante la actividad física (AF) a beneficios de tipo físico, psicológico y cognitivo (American Collegue of Sports Medicine, 2020; Chen et al., 2020).

A nivel psicológico la AF ha demostrado efectos positivos en adolescentes, observándose una relación inversa con la depresión (Bailey et al., 2018), el estrés (Nguyen-Michel et al., 2006), la ansiedad (Anderson \& Shivakumar, 2013) o la mejora de la sensación de bienestar (Hosker et al., 2019) y de vida plena (Águila Soto \& López Vargas, 2019). A nivel físico, muchos estudios han evidenciado los efectos de la inactividad física en la mortalidad (Kokkinos et al., 2011), diabetes (Wang et al., 2017), problemas cardiovasculares o en enfermedades no transmisibles (WHO, 2009) y una mejora de la condición física saludable del alumnado (Pastor-Vicedo et al., 2015). Por último, la práctica de AF preserva el desarrollo de diferentes variables cognitivas (Álvarez-Bueno et al., 2017; Chaddock et al., 2010; Donnelly et al., 2016; Erickson et al., 2009), por lo tanto, durante la cuarentena la EF puede producir mejoras en el rendimiento académico (Dwyer, Sallis, Blizzard, Lazarus \& Dean, 2001; Maureira, Díaz, Foos, Ibañez, Molina, Aravena, et al., 2014).

Teniendo en cuenta el importante papel de la AF para afrontar el potencial problema psicológico y de salud derivado del periodo de cuarentena por COVID-19 (Wang et al., 2020) y específicamente el rol de la EF en este propósito, el dominio técnico de la enseñanza virtual es esencial. Los docentes de EF (DEF) muestran actitudes positivas hacia las 
Tecnologías de la Información y Comunicación (TIC), destacándolas como herramientas facilitadoras de la innovación pedagógica y el aprendizaje (Díaz-Barahona et al., 2019). A pesar de ello, los DEF no se sienten preparados para incorporar la tecnología en sus aulas (Gibbone et al., 2010) y algunos no valoran su importancia (Ramos et al., 2019), quizás porque la integración tecnológica en EF ha sido principalmente diseñada para su uso presencial en clase (Baek et al., 2018; Juniu, 2011).

De este modo, la modalidad no presencial podría ser un reto para algunos DEF, ya que el confinamiento ha supuesto la integración de las TIC de manera inmediata y obligatoria, proceso que se dificulta por el componente predominantemente motriz de los contenidos. En cuanto a los estudiantes, Carroll \& Morrell (2006) destacaron la importancia de desarrollar sus habilidades tecnológicas, idea que cobra importancia en cuarentena. En este contexto educativo especial, Chen et al. (2020) afirman que la tecnología es la única vía viable para mantener la función física y la salud mental durante el confinamiento. Por otro lado, las TIC también se requieren a nivel comunicativo y de gestión en los DEF (Hyeon et al., 2018; Kretschmann, 2015). Esto incluye la comunicación con el resto de estamentos educativos como otros profesores o los responsables legales (Carriedo et al., 2020). Por lo tanto, en cuarentena las TIC se requieren para educar y mejorar los niveles de AF en los alumnos, y también para un uso comunicativo y de gestión. Además, la intervención docente se puede ver afectada por la conciliación familiar (Burgess \& Sievertsen, 2020). No todos los DEF tendrán las mismas facilidades para ejercer la docencia virtual con normalidad y su disponibilidad estará condicionada por la situación personal.

Respecto al proceso evaluativo, podría ser uno de los principales problemas al que tiene que enfrentarse el profesorado durante la cuarentena en ausencia de tareas presenciales. Establecer un proceso de evaluación continua hace que la evaluación y la calificación puedan dificultarse. En cuanto a los contenidos de EF, los diferentes decretos autonómicos los organizan de forma general en torno a la salud, motricidad, el cuerpo como medio de expresión o la práctica de juegos y deportes, entre otros. Cada programación didáctica lo adapta según el curso y el instituto, sin embargo, dada la excepcionalidad del periodo de cuarentena se desconoce qué contenidos han sido posibles. El confinamiento podría haber condicionado los contenidos a impartir, ya sea porque el carácter virtual de la enseñanza imposibilita la realización de determinadas actividades, o bien, porque el profesorado ha priorizado aquellos contenidos más efectivos para mejorar la salud psicológica y física del alumnado. Por lo tanto, el objetivo del presente estudio es conocer y describir la intervención docente del profesorado de EF durante el confinamiento a nivel metodológico, evaluativo y sobre la reorganización de contenidos.

\section{Material y Métodos.}

\section{Participantes}

Se realizó un diseño de encuesta transversal para evaluar la integración de las acciones educativas a través de la tecnología en DEF durante la cuarentena por COVID-19. Los criterios de inclusión fueron ser profesor de EF, estar actualmente activo e inscribirse con un correo institucional-educativo de España.

\section{Procedimiento}

Los encuestados fueron invitados electrónicamente a través de la plataforma Google Forms. Se difundió a través de diferentes instituciones educativas y se alentó a compartirse con otros compañeros. Se firmaron los consentimientos informados. La recopilación de datos tuvo lugar durante cuatro semanas días (30 de marzo a 30 de abril de 2020). Se ofreció un periodo de adaptación de 15 días al profesorado después de que el Gobierno de España declara el periodo de cuarentena el 15 de marzo.

\section{Instrumento}

Se revisaron encuestas relacionadas con el uso de la tecnología en docentes de EF. No se encontró ninguna encuesta centrada en evaluar el uso de la tecnología en DEF para la docencia virtual. Por lo tanto, el diseño del cuestionario fue de elaboración propia. Se recopilaron datos sociodemográficos incluyendo género, edad y curso académico impartido durante el periodo de cuarentena. El cuestionario se estructuró en quince preguntas que abordaron las tres dimensiones estudiadas (metodología, evaluativa y contenidos) así como preguntas sobre los efectos físicos y psicológicos que podría producir la cuarentena en el alumnado. En cuatro de las preguntas del cuestionario se utilizó un tipo de escala Likert de cinco puntos. También se utilizaron preguntas de elección simple y de dos vías. El cuestionario fue elaborado utilizando un lenguaje entendible, con formulación neutra y evitando la ambigüedad.

\section{Análisis estadístico}

Las estadísticas descriptivas se calcularon para las características demográficas, el curso académico y las cuestiones analizadas. Se realizó un análisis de tipo cuantitativo. Los porcentajes mostrados se calcularon de acuerdo con el número de encuestados por respuesta con respecto al número total. Los resultados se expresaron como porcentajes. El análisis estadístico se realizó utilizando SPSS Statistic 21.0 (IBM SPSS Statistics, Nueva York, Estados Unidos).

\section{Resultados}

\section{Encuestados}

Los resultados iniciales presentaron las variadas formas de intervención docente durante el periodo de cuarentena por COVID-19 de una muestra de docentes de EF en España. El cuestionario fue realizado por 191 docentes (133 varones y 58 mujeres), de los cuales tres no estaban en activo durante el periodo de cuarentena (tasa de finalización: 98,4\%). Debido a las situaciones particulares de cada docente, algunas de las preguntas no fueron contestadas por todos los encuestados.

En la figura 1, se reflejan las franjas de edad de los docentes encuestados, observándose la mayoría de participación entre los 31 y 40 años.

62 de los encuestados impartían docencia en el primer ciclo de primaria $\left(1^{\circ}\right.$ y $2^{\circ}$ de primaria $), 64$ en el segundo ciclo 


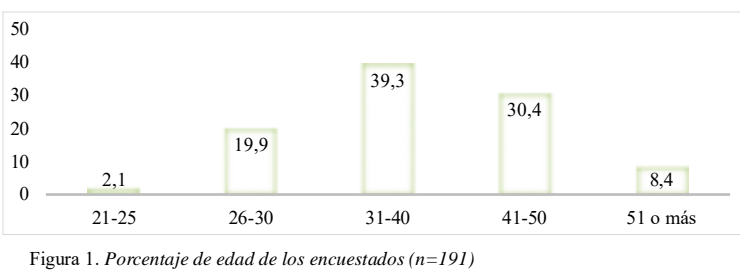

( $3^{\circ}$ y $4^{\circ}$ de primaria) y 59 en el tercer ciclo $\left(5^{\circ}\right.$ y $6^{\circ}$ de primaria $)$. Con respecto a los docentes de secundaria, 91 impartía en el primer ciclo $\left(1^{\circ}\right.$ y $2^{\circ}$ de secundaria), 82 en el segundo ciclo ( $3^{\circ}$ y $4^{\circ}$ de secundaria) y 57 en bachillerato.

\section{Organización de las tareas}

Los resultados relacionados con la realización de las tareas durante el periodo de cuarentena, han sido organizados alrededor de tres factores (tabla 1). Respecto al envío de tareas al alumnado durante el confinamiento por COVID-19, el 95,5\% indicó realizar algún tipo de tarea. Del profesorado que no había realizado ninguna actividad, el 68,75\% indicó que debían de empezar a hacerlo, mientras que el 31,25\% restante confirmó no poder hacerlo por motivos como la conciliación familiar, falta de conocimiento o por no considerarlo necesario.

En cuanto a la regularidad con la que el profesorado establecía contacto con el alumnado para el envío de tareas, un $64,5 \%$ lo hizo semanalmente. Un 19,9\% de los encuestados envió un trabajo general para todo el proceso de cuarentena. En el caso del profesorado que mantuvo la labor docente en los mismos días y horas previos a la cuarentena fue del $14 \%$. Finalmente, el 1,6\% no envió ninguna actividad.

En relación al seguimiento de las tareas, el 38,8\% únicamente hizo un seguimiento de las actividades en algunos casos. El 31,7\% lo hizo utilizando medios tecnológicos como videoconferencias y un $13,7 \%$ a través de los responsables legales del alumnado. Por último, el 15,8\% indicó no haber realizado seguimiento de las actividades enviadas.

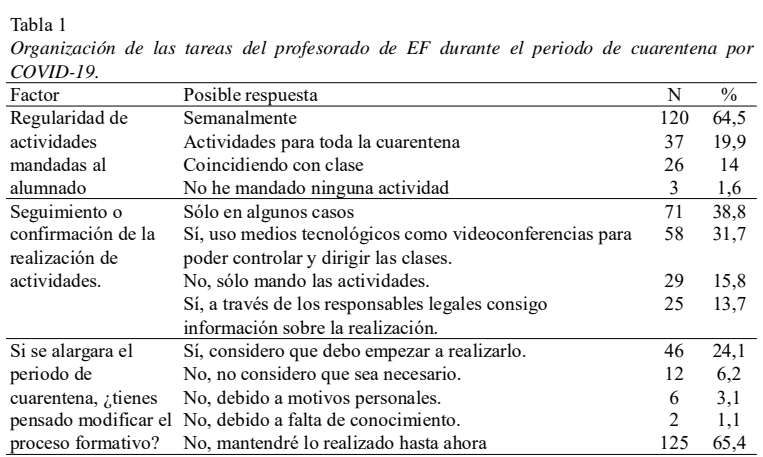

\section{les}

\section{Comunicación con el alumnado-representantes lega-}

Los resultados reflejaron como el $67,8 \%$ se comunicó a través de plataformas virtuales como Moodle, Google Classroom y otras similares. Un 13,7\% lo hizo a través de correo electrónico y un $18,5 \%$ mediante otros medios diferentes a los anteriores. El 41,8\% indicó haber hecho uso con regularidad de las redes sociales para transmitir actividades o tareas mientras que el $58,3 \%$ restante indicó no hacerlo en ningún caso o raras veces (Tabla 2)
Tabla 2

Medios de comunicación usados por el profesorado de EF durante el periodo de cuarentena por COVID-19.

\begin{tabular}{|c|c|c|}
\hline Factor & Posible respuesta & \\
\hline \multirow[t]{2}{*}{$\begin{array}{l}\text { Vías de comunicación para notificar } \\
\text { actividades a realizar }\end{array}$} & $\begin{array}{l}\text { Plataforma virtual (Moodle, Google Classroom u } \\
\text { otras) }\end{array}$ & $\begin{array}{l}12 \\
4\end{array} 67,8$ \\
\hline & Correo electrónico del padre/madre o tutor/a. & \\
\hline \multirow{3}{*}{$\begin{array}{l}\text { ¿Has hecho uso de las redes sociales } \\
\text { para poder transmitir actividades o } \\
\text { tareas? }\end{array}$} & Sí, a menudo & 764 \\
\hline & $\mathrm{n}$ caso & 723 \\
\hline & No, rara vez & 34 \\
\hline
\end{tabular}

\section{Distribución de contenidos}

La tabla 3 muestra los datos obtenidos sobre los contenidos desarrollados. En relación al primer tipo de actividades, las relacionadas con el cuerpo y sus habilidades perceptivo-motrices o cualidades motrices, los datos reflejan una puntuación mayoritaria entre 3 y 4 del $66,1 \%$. Con respecto a las tareas sobre salud y condición física, cabe destacar que un $20,8 \%$ indicó que todas las actividades enviadas tenían relación con este bloque. Por el contrario, enviaron en menor medida actividades relacionadas con expresión corporal y motriz (el 22,5\% indicaba no haber enviado ninguna actividad sobre esta temática) y sobre juego y deporte (el 35,9\% le daba una puntuación de 1 o 2).

\begin{tabular}{llcc}
$\begin{array}{l}\text { Tabla } 3 \\
\text { Porcentaje de actividades realizadas atendiendo a bloques de contenidos. }\end{array}$ & & \\
\hline Factor & Posible respuesta & $\mathrm{N}$ & $\%$ \\
\hline "El cuerpo y sus habilidades perceptivo & (1) Casi ninguna & 19 & 10,4 \\
motrices o cualidades motrices". & (2) Poco & 19 & 10,4 \\
& (3) Un uso medio & 76 & 41,5 \\
& (4) Un uso amplio & 45 & 24,6 \\
& (5) Muy abundante & 24 & 13,1 \\
\hline "Salud y condición fisica". & (1) Casi ninguna & 7 & 3,8 \\
& (2) Poco & 17 & 9,3 \\
& (3) Un uso medio & 60 & 32,8 \\
& (4) Un uso amplio & 61 & 33,3 \\
& (5) Muy abundante & 38 & 20,8 \\
\hline "Expresión corporal: Expresión y creación & (1) Casi ninguna & 41 & 22,5 \\
& (2) Poco & 26 & 14,3 \\
artística motriz". & (3) Un uso medio & 55 & 30,2 \\
& (4) Un uso amplio & 35 & 19,2 \\
& (5) Muy abundante & 25 & 13,7 \\
\hline "El juego y el deporte". & (1) Casi ninguna & 29 & 16 \\
& (2) Poco & 36 & 19,9 \\
& (3) Un uso medio & 48 & 26,5 \\
& (4) Un uso amplio & 47 & 26 \\
& (5) Muy abundante & 21 & 11,6 \\
\hline & & &
\end{tabular}

\section{Reajuste evaluativo}

Con respecto a la evaluación y calificación durante el periodo de cuarentena se registraron resultados similares entre los que realizarían un proceso evaluativo constante (el $37 \%$ ) y los que indicaron haberlo pensado, pero no haber realizado ninguna actividad evaluativa en el momento de la encuesta (el 29,1\%). Un total de 37 docentes (que corresponde al 19,6\% que respondieron esta pregunta), reconocieron no haber pensado cómo evaluar a su alumnado cuando realizaron la encuesta y el 14,3\% no evaluaría (Tabla 4).

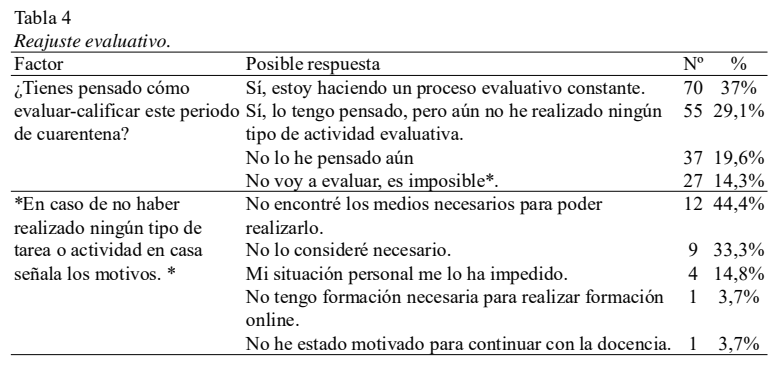

\section{Consecuencias psico-sociales}

Analizando las consecuencias psicosociales, los datos obtenidos son mostrados en la tabla 5. En relación a la cues- 
tión de si creían que el periodo de cuarentena podía afectarle a la salud física de su alumnado, el 56,8\% indicó que «sí, pero que a unos más que a otros». El 33,2\% opinaba que con seguridad les afectaría y un $8,4 \%$ consideraba que en principio no contemplaban esa opción o que en ningún caso consideraban que les afectaría $(1,6 \%)$.

Sobre si podía afectarle a su salud psicológica, se muestra que el 93,1\% consideraban que sí lo haría. Concretamente el $60,8 \%$ indicaba que sería más a unos que a otros y el 32,3\% lo afirmaron con seguridad. El porcentaje de encuestados que opinaba que no les influenciaría en su salud psicológica es menor que los que pensaban que no les afectaría a su salud física. Concretamente el 5,3\% indicaba no contemplarlo y un $1,6 \%$ decía que en ningún caso les perjudicaría.

Acerca de si pensaban que la práctica de AF podría ayudar a su alumnado a que el periodo de cuarentena del COVID19 fuera más ameno, una amplia mayoría $(96,3 \%)$ indicó que con seguridad les ayudaría. Ninguno de los encuestados consideró que «en cualquier caso» pudiera ayudar realizar deporte o actividad física a ser más ameno el periodo de cuarentena.

\begin{tabular}{llll}
$\begin{array}{l}\text { Tabla 5: } \\
\text { Consecuencias psicosociales. }\end{array}$ & & & \\
\hline Factor & Posible respuesta & N & $\%$ \\
\hline ¿Crees que el periodo de cuarentena & Sí, pero a unos más que a otros. & 108 & 56,8 \\
puede afectar a la salud fisica de tu & Ś, con seguridad. & 63 & 33,2 \\
alumnado? & No en principio no contemplo esa opción. & 16 & 8,4 \\
& No, en ningún caso. & 3 & 1,6 \\
\hline ¿Crees que el periodo de cuarentena & Sí, pero a unos más que a otros. & 115 & 60,8 \\
puede afectar a la salud psicológica & Sí, con seguridad. & 61 & 32,3 \\
de tu alumnado? & No en principio no contemplo esa opción. & 10 & 5,3 \\
& No, en ningún caso. & 3 & 1,6 \\
\hline ¿Consideras que la práctica de & Sí, con seguridad. & 183 & 96,3 \\
actividad física podría ayudar al & Tengo mis dudas & 6 & 3,2 \\
periodo de cuarentena a ser más & No creo que afecte demasiado & 1 & 0,5 \\
ameno? & No, en ningún caso. & 0 & 0 \\
\hline
\end{tabular}

\section{Discusión}

El presente estudio tenía como objetivo conocer como el DEF se ha adaptado la intervención docente a nivel metodológico, evaluativo y de la enseñanza de sus contenidos durante el periodo de cuarentena por COVID-19. Los resultados obtenidos ayudan a conocer las principales herramientas y estrategias utilizadas por los docentes y así reconocer posibles debilidades metodológicas en la enseñanza online por parte de los DEF. Asimismo indican como la mayoría de los docentes han optado por seguir la enseñanza online principalmente centrados en contenidos de carácter de condición física y salud, siendo la evaluación el aspecto que más controversia y dificultades ha generado al respecto.

El cuestionario realizado señala que el 95,5\% de los DEF ha realizado actividades durante el periodo de cuarentena, de los cuales aproximadamente el $65 \%$ ha decidido organizar el trabajo con frecuencia semanal. Estos datos indican como la gran parte de los DEF no han continuado en su horario habitual de clase, siendo solo el 14\% los que sí han mantenido la organización previa la cuarentena. Estos resultados podrían explicarse por barreras como la falta de conciliación familiar o por la diversidad de dificultades tecnológicas encontradas que impiden hacer frente a la rutina previamente establecida (Burgess \& Sievertsen, 2020). De esta forma, entre los obstáculos identificados a la hora de implementar recursos tecnológicos en las clases de EF, Villalba et al., (2017) destacan principalmente la reducción del tiempo dedicado a la realización de AF o la falta de recursos tecnológicos. Estos mismos autores señalan adicionalmente, como algunos docentes hacen énfasis en reconocer su falta de conocimiento o la presencia de problemas técnicos, aspectos que coinciden con nuestros resultados, nuestros resultados indican que solo el $1 \%$ lo atribuye a este factor.

En esta restructuración metodológica durante la cuarentena, el proceso evaluativo es uno de los grandes retos al que tiene que enfrentarse el profesorado (Burgess \& Sievertsen, 2020). La imposibilidad de realizar tareas presenciales, así como establecer un proceso de evaluación continua hacen que la evaluación y la calificación durante el periodo de cuarentena sea especialmente compleja. Este problema podría agravarse en los DEF como consecuencia del carácter motriz de los contenidos. De hecho, el 14,3\% asegura que no va a realizar evaluación porque lo considera imposible y el $20 \%$ aún no lo ha pensado. Este dato es preocupante debido a que la evaluación es una de las obligaciones curriculares y administrativas del docente. Por otro lado, el $67 \%$ si va a realizar evaluación, de hecho, aproximadamente la mitad de ese porcentaje considera que es un proceso que ya tiene sistematizado y preparado.

Más allá del mero aspecto calificativo, el objetivo primario de la evaluación es el de proporcionar información sobre el progreso del alumnado, tanto a los familiares como a los demás docentes. Las potenciales carencias informativas durante este proceso podrían retrasar la identificación de las dificultades de aprendizaje, pudiendo ocasionar consecuencias perjudiciales a largo plazo para el niño Andersen \& Nielsen (2019). Algunos autores han sugerido la realización de calificaciones pronosticadas si se cancela el proceso evaluativo, sin embargo, Murphy \& Wyness (2020) muestran que a menudo son inexactas, y que adicionalmente producen una mayor diferencia entre los estudiantes de alto rendimiento con aquellos de entornos desfavorecidos.

En el proceso evaluativo, la comunicación constante del DEF con el alumnado y los responsables legales es esencial. Durante la pandemia por COVID-19, el uso forzoso de medios tecnológicos con una finalidad comunicativa ha implicado el uso de diferentes plataformas y vías telemáticas. El $67,8 \%$ de los encuestados han optado por el uso de plataformas virtuales para establecer la comunicación docente, utilizando los restantes otras vías como correo electrónico y aplicaciones de comunicación interactiva móvil, entre otros.

Las redes sociales parecen ser una vía interesante para mejorar la interacción con el alumnado. La facilidad de acceso a la información a través de los teléfonos móviles, sumado a la familiarización en el manejo de las redes sociales por parte de los alumnos, podría suponer un aumento en su motivación e interés (Carriedo et al., 2020). Los resultados indicaron que más de la mitad de los docentes no ha usado este tipo de vías para comunicarse con el alumnado. Sin embargo, la mayoría de los adolescentes (95\%) tiene acceso a tecnología inteligente como teléfonos móviles, reconociendo que el $45 \%$ asegura estar en línea casi constantemente (Anderson \& Jiang, 2018). Por lo tanto, la tendencia educativa es aumentar los recursos tecnológicos para mejorar la comunicación entre el alumnado, responsables legales y los docentes, aunque el uso de las redes sociales como herramienta educativa parece no estar tan asentado por partes de los docentes encuestados. 
Diversas investigaciones consideran las TIC como un recurso esencial para las clases de EF (Browne, 2015; Hill \& Váldez-García, 2020; O’Neil \& Krause, 2019), sin embargo la mayoría de los DEF no suelen usar tecnología en clase (Kretschmann, 2015). Ptomey et al. (2015), destaca como la tecnología ayuda al seguimiento de aspectos fundamentales como la nutrición y la realización de actividad física, contenidos de índole saludable que son competencia de la EF. Además, el uso de la TIC en las clases de EF se ha vinculado con mejoras en aptitudes cognitivas y la motivación (Legrain \& Gillet, 2015), y el rendimiento motor y los niveles de AF en el aula (Legrain \& Gillet, 2015; Melton, 2015). Un problema adicional, es que la integración tecnológica en EF ha sido estudiada para su uso en el gimnasio (Baek et al., 2018; Juniu, 2011). Estudios previos han analizado la integración la tecnología en EF para poder practicar desde casa, sugiriendo el empleo de las tabs-app como un recurso útil (Browne, 2015), los podscast para la enseñanza de contenidos teóricos (McNeill \&Fry, 2012), videos y multimedias de apoyo a la enseñanza (Tondeur et al., 2012), medidas digitales de ejercicio y rastreadores de la frecuencia cardíaca (Browne, 2015) e incluso el uso de redes sociales para fomentar el aprendizaje informal (Anderson \& Jiang, 2018). Por lo tanto, en el periodo de cuarentena se sugiere el uso de las TIC no solo para mejorar los niveles de AF en los alumnos sino también un importante uso comunicativo y de gestión del aula.

A pesar de las ventajas de la integración de las TIC en EF, muchos docentes no se sienten preparados para incorporar la tecnología a sus aulas de manera inmediata (Gibbone et al., 2010) e incluso algunos no valoran su importancia (Ramos et al., 2019). Varias investigaciones han destacado que los DEF reconocen su falta de conocimiento para aplicar las TIC (Baek et al., 2018; Gibbone et al., 2010; Juniu \& Shonfeld, 2013). Parece indicarse que cuanto más alto era el nivel de alfabetización informática del profesor, más probabilidades tenían de incluir la tecnología en EF (Kretschmann, 2015). Sin embargo, incluso el profesorado tecnológicamente competente señala dificultades que le ha impedido la implementación de la tecnología en su enseñanza (Krause \& Lynch, 2018). Aproximadamente uno de cada tres DEF no considera tener suficiente apoyo tecnológico para integrar la tecnología en sus clases (Hill \& Valdez-García, 2020). Esto podría ser explicado por una serie de dificultades asociadas como la falta de tiempo, acceso, incentivos o conocimiento por parte de los DEF (Hill \& Valdez-García, 2020). Independientemente del apoyo tecnológico, la falta de tiempo para explorar la tecnología ha sido la dificultad más mencionada por los DEF (Baek et al., 2018). Finalmente, Ramos et al. (2019) destacan la limitada integración de las TIC en el currículo y el poco reconocimiento de los esfuerzos realizados por los DEF por parte de las instituciones educativas.

Respecto a una posible reorganización de los contenidos impartidos, no se han encontrado investigaciones previas que evalúen qué tipos de contenido son impartidos por el DEF de manera virtual. La excepcionalidad de la cuarentena y el carácter motriz de los contenidos hacen que este planteamiento no haya sido estudiado en profundidad. Los DEF encuestados señalaron que los contenidos relacionados con la condición física y la salud fueron los más trabajados, aproximadamente el 75\% destacó que haría entre un uso medio y abundante de este bloque contenido, confirmando un 21\% que harían un uso muy abundante de las misma. Por otro lado, en cuanto al bloque de contenidos de expresión corporal, cerca del $20 \%$ indicó que realiza muy pocas actividades virtuales de este contenido, resultado parecido al mostrado con los juegos y deportes donde aproximadamente uno de cada tres DEF especifica no realizar apenas actividades de este bloque.

Los resultados relativos a la distribución que se realizó de los contenidos pueden quedar explicados debido a haberse priorizado aquellos que producen un efecto más directo sobre la salud del alumnado. De hecho, la mayoría de las recomendaciones relacionadas con la práctica de AF durante la cuarentena para adolescentes están dirigidas a actividades del bloque de contenidos de condición física y salud (American College of Sports Medicine, 2020; WHO, 2020). Ejercicios con autocargas, utilizando utensilios domésticos como pesos o subir o bajar escaleras, han sido parte de las recomendaciones realizadas por las instituciones e investigadores (Chen et al., 2020a; Simpson et al., 2020). Sin embargo, muchas de estas recomendaciones también incluyen la realización de actividades expresivas como los bailes, con la finalidad de propiciar una activación cardiovascular.

Durante más de un mes y medio, los niños y adolescentes no han podido salir de casa, por lo que la posibilidad de la práctica de AF se podría ver reducida y aumentados los comportamientos obesogénicos. Según un informe reciente de los comportamientos saludables de los adolescentes y niños españoles, se muestra como más del $66 \%$ solo realiza ejercicio físico durante la hora de EF (PASOS, 2019).

Nuestra investigación muestra como aproximadamente el $90 \%$ de los docentes considera que su alumnado va a tener algún tipo de problema relacionado con la salud, haciendo énfasis en que uno de cada tres espera que la salud física de deteriore en todos sus alumnos sin excepción. En contra, el 10\% espera que no habrá demasiadas consecuencias físicas, y solo el 1,6 está seguro de que no habrá problemas físicos durante la cuarentena. Hay dos aspectos esenciales que pueden incidir en el aumento de conductas sedentarias en los niños y adolescentes. Por un lado, Emery et al. (2015) señala que las características de los hogares actuales propician el desarrollo de comportamientos que inducen al sedentarismo, como por ejemplo el acceso ilimitado a medios de entretenimiento pasivo.

Por otro lado, la pérdida de una rutina puede inducir a comportamientos obesogénicos como defiende la hipótesis de los días estructurados. En relación a esta idea, Brazendale et al. (2017), comparó los comportamientos en edad primaria dependiendo de los días laborables y los de fin de semana, teóricamente menos estructurados, evidenciado la aparición de comportamientos más sedentarios durante los periodos no lectivos.

Respecto a los potenciales problemas psicológicos, la ansiedad, el estrés o el miedo provocados por el COVID-19 han sido evidenciados (Wang et al., 2020). En el caso de los niños y adolescentes, este problema también podría presentarse (Wang et al., 2020). Estas afirmaciones, confirman nuestros resultados, ya que el $91 \%$ de los DEF espera algún tipo de problema psicológico en su alumnado, sugiriendo incluso un $32 \%$ que este problema afectará a todos los discentes. 
El efecto positivo de la AF sobre las variables psicológicas como la sensación de bienestar, relajación o disminución del estrés ha sido confirmado (García \& Froment, 2018; ReynagaEstrada et al., 2016). Adicionalmente, se ha evidenciado que si esta práctica de $\mathrm{AF}$ es en entornos naturales los efectos son mayores que si es realizada en entornos artificiales (Crone-Grant \& Grant, 2000; Lawton et al., 2017), condición que no ha sido posible durante el confinamiento.. Por lo tanto, parece indicarse que, aunque la mayoría de los DEF aseguran haber resuelto el problema de la realización diaria de AF, parecen no estar muy confiados en que sus propuestas sean suficientes para alcanzar beneficios a nivel físico y psicológico.

\section{Conclusiones}

La enseñanza de la EF durante la cuarentena por COVID19 ha supuesto una modificación de la intervención docente. Esta intervención, es especialmente importante debido a la repercusión de sus contenidos en la preservación de la salud física y mental durante el confinamiento. Los resultados han mostrado como los docentes de EF han priorizado la enseñanza de contenidos durante el confinamiento relacionados con el bloque de condición física y salud y relegando a un segundo lugar contenidos de expresión corporal, juegos y deportes o habilidades motrices. Estos contenidos han sido organizado prioritariamente a través de un desglose semanal de las actividades, sin seguir en gran parte el horario habitual previo y donde más de la mitad no va a realizar un seguimiento o comprobación del cumplimiento de las tareas.

Respecto al proceso evaluativo, se han detectado una gran incertidumbre, incluso algunos docentes no van a implementarlo. Solo la mitad de los docentes encuestados confirma que van a realizar una evaluación, siendo un tercio de estos, los que garantiza que lo tiene ya planificado y organizado.

Se han usado diferentes canales comunicativos con el alumnado o padres se ha realizado principalmente a través de plataformas virtuales y secundariamente a través de correo electrónico. Una minoría también reconoce el uso de mensajería instantánea móvil para comunicarse. En este sentido, las redes sociales han sido utilizada por la mayoría, siendo un tercio reacio a su uso.

Finalmente, la gran parte de los docentes consideran que el confinamiento va a producir algún tipo de problema físico y/o psicológico en su alumnado, siendo conscientes de la importancia que tiene la realización de AF durante la no asistencia a clase durante el confinamiento por COVID-19 y valorando casi su la totalidad, que la EF ha sido fundamental para aliviar este periodo.

\section{Referencias}

Águila, C. \& López, J. J. (2019). Cuerpo, corporeidad y educación: una mirada reflexiva desde la Educación Física. Retos: Nuevas Perspectivas de Educación Física, Deporte y Recreación, 2041(35), 413-421. Recuperado de: https:// recyt.fecyt.es/index.php/retos/article/view/62035

Álvarez-Bueno, C., Pesce, C., Cavero-Redondo, I., Sánchez-
López, M., Martínez-Hortelano, J.A., \& Martínez-Vizcaíno, V. (2017). The Effect of Physical Activity Interventions on Children's Cognition and Metacognition: A Systematic Review and Meta-Analysis. Journal of the American Academy of Child and Adolescent Psychiatry, 56(9), 729738. https://doi.org/10.1016/j.jaac.2017.06.012

American College of Sports Medicine. (2020). Keeping Children Active during the Coronavirus Pandemic. Recuperado de: https://www.exerciseismedicine.org/assets/ page_documents/EIM_Rx for Health_Keeping Children Active During Coronavirus Pandemic.pdf

American Collegue of Sports Medicine. (2020). The Coronavirus Pandemic. Recuperado de: https://www.acsm.org/readresearch/newsroom/news-releases/news-detail/2020/03/16/ staying-physically-active-during-covid-19-pandemic

Anderson, E., \& Shivakumar, G. (2013). Effects of exercise and physical activity on anxiety. Frontiers in Psychiatry, 4, 10 13.

https://doi.org/10.3389/fpsyt.2013.00027

Anderson, M., \& Jiang, J. (2018). Teens, social media \& technology 2018. Recuperado de: https:// www.pewresearch.org/internet/2018/05/31/teens-social-media-technology-2018/

Andersen, S.C. \& Nielsen, H.S. (2019). Learning from Performance Information. Journal of Public Administration Research and Theory. Recuperado de: https://psa.dk/papers/ NT_Paper_180917.pdf

Baek, J.H. Hones, E., Bulger, S., \& Taliaferro, A. (2018). Physical education teacher perceptions of technology-related learning experiences: Aqualitative investigation. Journal of Teaching in Physical Education, 37(2), 175-185. https://doi.org/ 10.1123/jpe.2017-0180

Bailey,A.P., Hetrick, S. E., Rosenbaum, S., Purcell, R., \& Parker,A. G. (2018). Treating depression with physical activity in adolescents and young adults: A systematic review and metaanalysis of randomised controlled trials. Psychological Medicine, 48(7), 1068-1083. https://doi.org/10.1017/ S0033291717002653

Berardelli, I., Corigliano, V., Hawkins, M., Comparelli,A., Erbuto, D., \& Pompili, M. (2018). Lifestyle interventions and prevention of suicide. Frontiers in Psychiatry, 9, 1-10. https:/ /doi.org/10.3389/fpsyt.2018.00567

Brazendale, K., Beets, M. W., Weaver, R. G., Pate, R. R., TurnerMcGrievy, G M., Kaczynski,A. T., Chandler, J. L., Bohnert, A., \& von Hippel, P. T. (2017). Understanding differences between summer vs. school obesogenic behaviors of children: The structured days hypothesis. International Journal of Behavioral Nutrition and Physical Activity, 14(1), 1-14.https://doi.org/10.1186/s12966-017-0555-2

Browne, T. (2015). A case study of student teachers' learning and perceptions when using tablet applications teaching physical education. Asia-Pacific Journal of Health, Sport and Physical Education, 6(1), 3-22. https://doi.org/10.1080/ 18377122.2014.997858

Burgess, S., \& Sievertsen, H. H. (2020). Schools, skills, and learning: The impact of COVID-19 on education. Recuperado de: https://voxeu.org/article/impact-covid-19-education

Carriedo,A., Méndez-Giménez,A., Fernández-Río, J., \& Cecchini, J. A. (2020). New ways and resources for teaching body expression in physical education: Internet and viral challenges 
[Nuevas posibilidades y recursos para la enseñanza de la expresión corporal en educación física: internet y los retos virales]. Retos, 37, 722-730. Recuperado de: https:// recyt.fecyt.es/index.php/retos/article/view/69147

Carroll, J. B., \& Morrell, P. D. (2006). A Comparison of Teacher Education Faculty and Preservice Teacher Technology Competence. Journal of Computing in Teacher Education, 23(1),5-10. https://doi.org/10.1080/10402454.2006.10784554

Chaddock, L., Erickson, K. I., Prakash, R. S., Kim, J. S., Voss, M. W., Vanpatter, M., Pontifex, M. B., Raine, L. B., Konkel,A., Hillman, C. H., Cohen, N. J., \& Kramer, A. F. (2010). A neuroimaging investigation of the association between aerobic fitness, hippocampal volume, and memory performance in preadolescent children. Brain Research, 1358, 172 183.https://doi.org/10.1016/j.brainres.2010.08.049

Chen, P., Mao, L., Nassis, G P., Harmer, P., Ainsworth, B. E., \& Li, F. (2020a). Wuhan coronavirus (2019-nCoV): The need to maintain regular physical activity while taking precautions. Journal of Sport and Health Science 9(2), 103-104. https:// doi.org/10.1016/j.jshs.2020.02.001

Chen, P., Mao, L., Nassis, G. P., Harmer, P., Ainsworth, B., \& Li, F. (2020b). Returning Chinese school-aged children and adolescents to physical activity in the wake of COVID-19: Actions and precautions. Journal of Sport and Health Science, 4-6. https://doi.org/10.1016/j.jshs.2020.04.003

Clark, H., Coll-Seck,A. M., Banerjee, A., Peterson, S., Dalglish, S. L., Ameratunga, S., Balabanova, D., Bhan, M. K., Bhutta, Z. A., Borrazzo, J., Claeson, M., Doherty, T., El-Jardali, F., George, A. S., Gichaga, A., Gram, L., Hipgrave, D. B., Kwamie, A., Meng,Q., Costello,A.(2020).Afuture for the world's children? A WHO-UNICEF-Lancet Commission. The Lancet, 395(10224), 605-658. https://doi.org/10.1016/S01406736(19)32540-1

Cluver, L., Lachman, J. M., Sherr, L., Wessels, I., Krug, E., Rakotomalala, S., Blight, S., Hillis, S., Bachman, G., Green, O., Butchart, A., Tomlinson, M., Ward, C. L., Doubt, J., \& McDonald, K. (2020). Parenting in a time of COVID-19. The Lancet, 2019(20), 30736. https://doi.org/10.1016/s01406736(20)30736-4,64

Crone-Grant, D., \& Grant, T. (2000). Physical activity and mental health. ALife in the Day, 4(4), 11-14. https://doi.org/10.1108/ 13666282200000029

Díaz-Barahona, J., Molina-García,J., \&Monfort-Pañego,M.(2019). Estudio de las actitudes y el interés de los docentes de primaria de educación física por las TIC en la Comunidad Valenciana. [Primary Physical Education teacher's attitudes and interests towards ICT in the Valencian Community]. Retos, 35(1), 267-272. Recuperado de: https://recyt.fecyt.es/ index.php/retos/article/view/63355/41417

Donnelly, J. E., Hillman, C. H., Castelli, D., Etnier, J. L., Lee, S., Tomporowski,P., Lambourne, K., \& Szabo-Reed,A. N.(2016). Physical activity, fitness, cognitive function, and academic achievement in children: Asystematic review. Medicine and Science in Sports and Exercise, 48(6), 1197-1222. https:// doi.org/10.1249/MSS.0000000000000901

Dwyer, T., Sallis, J., Blizzard, L., Lazarus, R. \& Dean, K. (2001). Relation of academic performance physical activity and fitness in children. Pediatric Exercise Science, 13, 225-237 https:// doi.org/10.1123/pes.13.3.225

Emery, C., Olson, K., Lee, V., Habash, J., \& Bodine, A. (2015).
Home environment and psychosocial predictors of obesity status among community-residing men and women CF. Int $J$ Obes, 39(9), 1401-1407. https://doi.org/10.1016/ j.physbeh.2017.03.040

Erickson, K. I., Prakash, R. S., Voss, M. W., Chaddock, L., Hu, L., Morris, K. S., White, S. M., Wójcicki, T. R., McAuley, E., \& Kramer, A. F. (2009). Aerobic fitness is associated with hippocampal volume in elderly humans. Hippocampus, 19(10), 1030-1039. https://doi.org/10.1002/hipo.20547

Fundación Gasol (2019). Informe Pasos. Recuperado de https:// www.gasolfoundation.org/wp-content/uploads/2019/11/Informe-PASOS-2019-online.pdf

García,A., \& Froment, F. (2018). Beneficios de la actividad física sobre la autoestima y la calidad de vida de personas mayores (Benefits of physical activity on self-esteem and quality of life of older. Recyt.Fecyt.Es, 2041(1986), 3-9. Recuperado de: http://revistas.unjbg.edu.pe/index.php/cs/article/view/ 499/445

Geniole, S. N., David, J. P. F., Euze, R. F. R., Toledo, B. Z. S., \& Neves, A. I. M. (2016). The Benefits of an Outdoor Walk Landfill Area Relative to Its Neighboring. 8(2), 107-120. https://doi.org/10.1089/eco.2016.0005

Gibbone, A., Rukavina, P., \& Silverman, S. (2010). Technology Integration in Secondary Physical Education: Teachers' Attitudes and Practice. Journal of Educational Technology Development and Exchange, 3(1), 27-42. https://doi.org/ 10.18785/jetde. 0301.03

Hill, G. M., \& Valdez-Garcia,A. (2020). Perceptions of Physical Education Teachers Regarding the Use of Technology in Their Classrooms. The Physical Educator, 77(1), 29-41. https://doi.org/10.18666/tpe-2020-v77-i1-9148

Hosker, D. K., Elkins, R. M., \& Potter, M. P. (2019). Promoting Mental Health and Wellness in Youth Through Physical Activity, Nutrition, and Sleep. Child and Adolescent Psychiatric Clinics of North America, 28(2), 171-193. https:/ /doi.org/10.1016/j.chc.2018.11.010

Huang, H., Fan, C., Li, M., Nie, H.-L., Wang, F.-B., Wang, H., Wang, R., Xia, J., Zheng, X., Zuo, X., \& Huang, J. (2020). COVID-19:ACall for Physical Scientists and Engineers. ACS Nano, 3747-3754. https://doi.org/10.1021/acsnano.0c02618 Hyeon, S., Reeve, J., \& Song, Y. (2018). Recommending goals and supporting needs/ : An intervention to help physical education teachers communicate their expectations while supporting students ' psychological needs Psychology of Sport \& Exercise Recommending goals and supporting needs/ : An intervention. Psychology of Sport \& Exercise, 41,107-118. https://doi.org/10.1016/j.psychsport.2018.12.008

Juniu, S. (2011). Pedagogical Uses of Technology in Physical Education. Journal of Physical Education, Recreation \& Dance, 82(9), 41-49. https://doi.org/10.1080/ 07303084.2011 .10598692

Juniu, S., \& Shonfeld, M. (2013). Technology integration in physical education teacher education programs/ : a comparative analysis. Actualidades investigativas en educación,13(3), https://doi.org/10.15517/aie.v13i3.12044

Kokkinos, P., Sheriff,H., \& Kheirbek, R. (2011). Physical inactivity and mortality risk. Cardiology Research and Practice, 1(1). https://doi.org/10.4061/2011/924945

Kopcha, T. J. (2012). Teachers' perceptions of the barriers to technology integration and practices with technology under 
situated professional development. Computers and Education, 59(4), 1109-1121. https://doi.org/10.1016/ j.compedu.2012.05.014

Krause, J. M., \& Lynch, B. M. (2018). Faculty and student perspectives of and experiences with TAFCK in EFTE. Curriculum Studies in Health and Physical Education, 9(1), 58-75.https://doi.org/10.1080/25742981.2018.1429146

Kretschmann, R. (2015). Effect of Physical Education Teachers' Computer Literacy on Technology Use in Physical Education. The Physical Educator, 72, 261-277. https://doi.org/ 10.18666/tpe-2015-v72-i5-4641

Lawton, E., Brymer, E., Clough, P., \& Denovan, A. (2017). The relationship between the physical activity environment, nature relatedness, anxiety, and the psychological well-being benefits of regular exercisers. Frontiers in Psychology, 8. https://doi.org/10.3389/fpsyg.2017.01058

Legrain, P., \& Gillet, N. (2015). Integration of Information and Communication Technology and Pupils ' Motivation in a Physical Education Setting. Journal of teaching in Physical Education,34(3), 384-401.https://doi.org/10.1123/jtpe.20140013

McNeill, M., \& Fry, J. (2012). The Value of ICT from a Learning Game-Playing Perspective. ICHEFR-SD Journal of Research, 7(2), 45-51. Recuperado de: https://files.eric.ed.gov/fulltext/ EJ993569.pdf

Melton, B. (2015). Evaluating a Physical Activity App in the Classroom: A Mixed Methodological Approach Among University Students. Physical Educator, 72(4), 601-620. December.https://doi.org/10.18666/TEF-2015-V72-I4-7139

Nguyen-Michel, S. T., Unger, J. B., Hamilton, J., \& Spruijt-Metz, D. (2006). Associations between physical activity and perceived stress/hassles in college students. Stress and Health, 22(3), 179-188. https://doi.org/10.1002/smi.1094

O’Neil, K., \& Krause, J. M. (2019). Physical Education Teacher Education Faculty Self-Efficacy Toward Educational Technology. The Physical Educator, 76(5), 1287-1305. https://doi.org/10.18666/tpe-2019-v76-i5-9107

Pastor-vicedo, J.C., Gil-madrona, P., Prieto-ayuso,A., \& Gonzálezvíllora, S. (2015). Los contenidos de salud en el área de educación física: Análisis del currículum vigente. RETOS. Nuevas Tendencias En Educación Física, Deporte y Recreación, 2041(28), 134-140. Recuperado de: https:// recyt.fecyt.es/index.php/retos/article/view/34900

Ptomey, L. T., Sullivan, D. K., Lee, J., Goetz, J. R., Gibson, C., \& Donnelly, J. E. (2015). The use of technology for delivering a weight loss program for adolescents with intellectual and developmental disabilities. Journal of the Academy of Nutrition and Dietetics, 115(1), 112-118. https://doi.org/ 10.1016/j.jand.2014.08.031

Ramos, C., Francisco, J., Vásquez, Á., Alejandra, P., Alvarez,A., Ramos, C., Francisco, J., Vásquez, Á., Alejandra, P., Alvarez, A., \& Alejandro, P. (2019). Barriers to ICT integration in interdisciplinary articulation from physical education. Journal of Sport and Health Research. 11(Supl 2):1-12.

Reynaga-Estrada, P., Vázquez, E. I. A., Gáleas, Á. M. V., Ortega, I. M. J., Serrano, M. D. L. P., \&Acosta, J. J. M. (2016). Beneficios psicológicos de la actividad física en el trabajo de un centro educativo. Retos, 2041(30), 203-206. Recuperado de: https://recyt.fecyt.es/index.php/retos/article/view/50254

Richard Murphy \& Gill Wyness. (2020). Minority Report: the impact of predicted grades on university admissions of disadvantaged groups, CEPEO Working Paper Series 20-07. Centre for Education Policy and Equalising Opportunities, UCL Institute of Education, revised Mar 2020. Recuperado de: https://repec-cepeo.ucl.ac.uk/cepeow/cepeowp20-07.pdf Simpson, R. J., Campbell, J. P., Gleeson, M., Krüger, K., Nieman, D. C., Pyne, D. B., Turner, E., \& Walsh, N. P. (2020). Can exercise affect immune function to increase susceptibility to infection? Exercise immunology review, 26, 8-22. Recuperado de: https://pubmed.ncbi.nlm.nih.gov/32139352/

Starkey, L. (2020). A review of research exploring teacher preparation for the digital age. Cambridge Journal of Education, 50(1), 37-56. https://doi.org/10.1080/ 0305764X.2019.1625867

Tondeur, J., Braak, J. Van, Sang, G., Voogt, J., Fisser, P., \& Ottenbreitleftwich,A. (2012). Preparing pre-service teachers to integrate technology in education/: A synthesis of qualitative evidence Computers \& Education Preparing pre-service teachers to integrate technology in education/: A synthesis of qualitative evidence. Computers \& Education, 1(11). https://doi.org/ 10.1016/j.compedu.2011.10.009

Villalba,A., González-Rivera, M. D., \& Díaz-Pulido, B. (2017). Obstacles perceived by physical education teachers to integrating ICT. Turkish Online Journal of Educational Technology, 16(1), 83-92. Recuperado de: https://eric.ed.gov/ ?id=EJ1124906

Wang, C., Pan, R., Wan, X., Tan, Y., Xu, L., Ho, C. S., \& Ho, R. C. (2020). Immediate psychological responses and associated factors during the initial stage of the 2019 coronavirus disease (COVID-19) epidemic among the general population in China. International Journal of Environmental Research and Public Health, 17(5). https://doi.org/10.3390/ijerph17051729

Wang, G.,Zhang, Y., Zhao, J., Zhang, J., \& Jiang, F.(2020). Mitigate the effects of home confinement on children during the COVID-19 outbreak. The Lancet,395(10228), 945-947.https:/ /doi.org/10.1016/S0140-6736(20)30547-X

Wang, X., Strizich, G., Hua, S., Sotres-Alvarez, D., Buelna, C., Gallo,L.C., Gellman, M.D., Mossavar-Rahmani,Y., O’Brien, M. J., Stoutenberg, M., Wang, T., Larissa Avilés-Santa, M., Kaplan, R.C.,\&Qi,Q.(2017). Objectively measured sedentary time and cardiovascular risk factor control in US Hispanics/ Latinos with diabetes mellitus: Results from the Hispanic Community Health Study/Study of Latinos (HCHS/SOL). Journal of the American Heart Association, 6(6). https:// doi.org/10.1161/JAHA.116.004324

WHO. (2009). Global Health Risks. Recuperado de: http:// www.who.int/healthinfo/global_burden_disease/ GlobalHealthRisks_report_full.pdf

WHO. (2020). Be Active during COVID-19. Recuperado de: https://www.who.int/news-room/q-a-detail/be-active-duringcovid-19

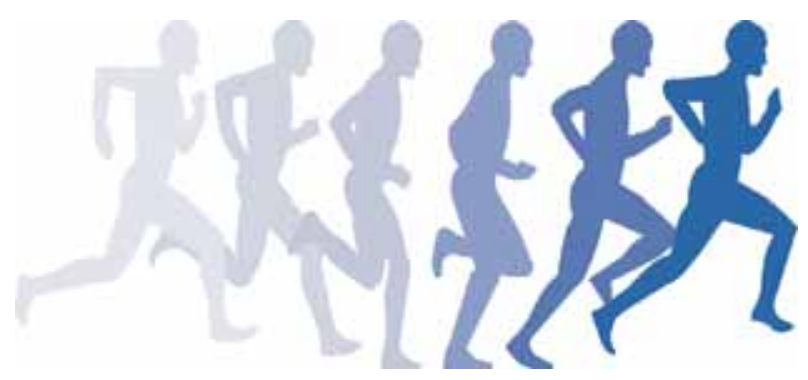

